\title{
Cardiac impact of high-frequency irreversible electroporation using an asymmetrical waveform on liver in vivo
}

Jing $\mathrm{Li}^{1,2+}$, Jingjing Wang ${ }^{3 \dagger}$, Xiaobo Zhang ${ }^{1}$, Xiao Zhang ${ }^{1}$, Hongmei Gao ${ }^{3 *}$ and Yueyong Xiao ${ }^{{ }^{*}}$

\begin{abstract}
Background: High-Frequency Irreversible Electroporation (H-FIRE) is a novel technology for non-thermal ablation. Different from Irreversible electroporation (IRE), H-FIRE delivers bipolar electrical pulses without muscle contraction and does not cause electrolysis. Currently, little is known regarding the cardiac safety during the administration of H-FIRE on liver. The aim of this study was to evaluate the changes of electrocardiogram (ECG) and biomarkers of cardiac damage during asymmetrical waveform of H-FIRE therapy in vivo.

Methods: The swines $(n=7)$ in IRE group, which used 100 pulses (2200 V, 100-100 $\mu$ s configuration), were administrated with muscle relaxant under anesthesia. In the absence of muscle relaxant, 7 swines in H-FIRE group were

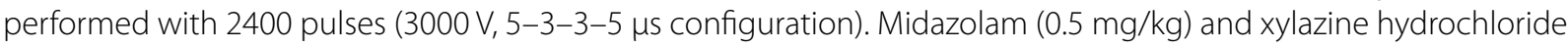
(20 mg/kg) were given to induce sedation, followed by Isoflurane (2.5\%, 100\% oxygen, $3 \mathrm{~L} / \mathrm{min}$ ) to maintain sedation in all the swines. Limb lead ECG recordings were analyzed by two electrophysiologists to judge the arrhythmia. Cardiac and liver tissue was examined by pathology technique.
\end{abstract}

Results: The ablation zones were larger in H-FIRE than IRE. Both IRE and H-FIRE did not affect the autonomous cardiac rhythm. Even when the electrical signal of IRE and H-FIRE fell on ventricular vulnerable period. Moreover, cTnl in IRE group showed an increase in $4 \mathrm{~h}$ after ablation, and decreased to baseline $72 \mathrm{~h}$ after ablation. However, cTnl showed no significant change during the administration of H-FIRE.

Conclusions: The study suggests an asymmetrical waveform for H-FIRE is a promising measure for liver ablation. The results were based on normal liver and the swines without potential cardiac diseases. With the limitations of these facts, asymmetrical waveform for H-FIRE of liver tissue seems relatively safe without major cardiac complications. The safety of asymmetrical waveform for H-FIRE needs to evaluate in future.

Keywords: High-frequency irreversible electroporation, Irreversible electroporation, Liver ablation, Arrhythmia, Cardiac injury

*Correspondence: hmg20192021@163.com; xiaoyy19820130@163.com These authors contributed equally: Jing Li and Jingjing Wang

${ }^{1}$ Department of Radiology, The First Medical Center of Chinese, PLA General Hospital, Beijing, China

${ }^{3}$ Department of Critical Care Medicine, Tianjin First Center Hospital, Tianjin 300192, China

Full list of author information is available at the end of the article

\begin{abstract}
Background
Irreversible electroporation (IRE) is a non-thermal ablation technology that creating irreversible nanoscale pores in the cell membrane and leading to cell death through apoptosis [1]. Based on the application of high voltage and short monopolar pulses with two electrodes placed across the target site, IRE has been implemented to ablate primary and metastatic hepatic or pancreatic tumors
\end{abstract}


near thermosensitive structures. IRE was known to cause muscle contractions during treatment. Sedative and neuroparalytic agents should be used in patients undergoing invasive mechanical ventilation [2]. Moreover, IRE adhere to the heart can be cardiac arrhythmia when the electrical pulse delivered outside of the absolute refractory period of the cardiac cycle $[3,4]$. Thus, synchronization was recommended to use during the operation of IRE in case of fatal cardiac arrhythmia $[5,6]$.

High-Frequency Irreversible Electroporation (H-FIRE) is the next-generation nanoknife ablation technology to conquer many of the technical barriers of conventional IRE as mentioned above [7]. Previous studies have showed that H-FIRE, which delivered ultra-short bipolar electrical pulses, minimized the stimulation of nerve and muscle. There is widespread speculation that H-FIRE could be used without ECG monitoring and taking no account of synchronization with $\mathrm{R}$ wave on ECG [8]. However, it is well-known that the principle of electroporation is permeabilization of the cell membrane around the electrode. The pore produced by electroporation affected the transportation of ion across the cell membrane. In reality, whether H-FIRE affects the cardiac electrophysiology is still unknown.

There are two wave-forms of H-FIRE. In theory, asymmetric waveform is more effective than symmetrical waveform [9]. Es et al. had demonstrated an asymmetric waveform for H-FIRE creates deeper lesions than symmetric waveform of the same energy and frequency [10]. However, the cardiac safety did not evaluate. The purpose of the present study is to evaluate cardiac safety in vivo with asymmetric waveform of H-FIRE in contrast to the conventional IRE.

\section{Methods}

\section{Animals}

All animal experimental protocols were approved by the Institutional Animal Care and Use Committee of The General Hospital of People's Liberation Army (License Number: 2019-D15-07). The study was conformed to the National Institutes of Health Guide for Animal Care and Use of Laboratory Animals (NIH Publication No. 85-23, revised 2011). Female Ba-Ma miniature pigs weighed 20-25 kg were used for this study (Shi-chuang Animal Labs, Beijing, China).

\section{Surgical procedures}

Experiments were carried out with midazolam $(0.5 \mathrm{mg} /$ $\mathrm{kg})$ and ketamine $(10 \mathrm{mg} / \mathrm{kg})$ for induction and Isoflurane inhalation (1-3\%) for maintenance. All the swines were under intubation and mechanical ventilation. Animals were continuously monitored using telemetry and pulse oximetry. Of the 14 animals, 7 animals underwent
IRE, the remaining 7 animals underwent H-FIRE. Before delivery of the IRE, the swines received vecuronium $(0.05-0.1 \mathrm{mg} / \mathrm{kg})$ to limit muscle contraction. All the animals were sacrificed within $72 \mathrm{~h}$ of the procedure. An overdose of pentobarbital sodium $(150 \mathrm{mg} / \mathrm{kg})$ was used to sacrifice all the animals.

The upper midline incision $(15-20 \mathrm{~cm})$ was made and the liver was exposed. Subsequently, the two platinumiridium electrodes of IRE or H-FIRE were positioned in parallel with spacing set to $1.5 \mathrm{~cm}$ on the lateral segment of the liver, and the active portion of the electrodes exposed to a length of $1.5 \mathrm{~cm}$. The electrodes were inserted into the hepatic parenchyma to a final depth of $2.5-3.0 \mathrm{~cm}$ (Fig. 1).

\section{IRE and H-FIRE protocol}

IRE (YTL-GM01, Inteligent Health, Tianjin) delivery was using computerized algorithms and square pulses $2200 \mathrm{~V}$ was delivered without $R$ wave synchronization. Each IRE treatment group consisted of 10 pulses (total 10 groups and 100 pulses), each pulse sustained 100 microseconds with a 1000 ms pause between each group of pulses (Fig. 2, Table 1).

H-FIRE was generated by a custom-built pulse generation system (Inteligent Health, Tianjin) with the ability to deliver microsecond, biopolar pulses in rapid bursts. The positive voltage presents pulse width of $5 \mu \mathrm{s}$, with a delay of $3 \mu$ s between each change in polarity. The following negative voltage presents pulse width of $3 \mu \mathrm{s}$, with a delay of $5 \mu$ s between each change in polarity (Fig. 2). A total of 2400 bursts at a $3000 \mathrm{~V}$ amplitude was set per sequence, and with a 1000 ms pause between each group of pulses (Table 1).

\section{Cardiac monitoring}

Cardiac rhythms was recorded for each procedure with a single-lead ECG (XD-7100, Tai yi, Shang hai, China). Animals were divided into two groups underwent 100 or 2400 bursts of liver ablations using IRE or H-FIRE, respectively. These ECG rhythms were analyzed by two electrophysiologists back to back. An ECG was recorded every $5 \mathrm{~min}$ for up to $30 \mathrm{~min}$ after ablation. Major cardiac complications are defined as ventricular fibrillation, ventricular tachycardia, high degree Atrio-Ventricular blocks (AVB).

Two venous access were obtained on each sides of the groins using $8 \mathrm{~F}$ introducers. The biomarker of cardiac injury were obtained from the blood samples before IRE or H-FIRE intervention, $4 \mathrm{~h}$ and $72 \mathrm{~h}$ after IRE or H-FIRE intervention. Cardiac Troponin I (cTnI, normal range 0-1.68 ng/L, cTnI assay on Dimension Vista 1500 system, Siemens Healthineers, Erlangen, Germany) was detected. 

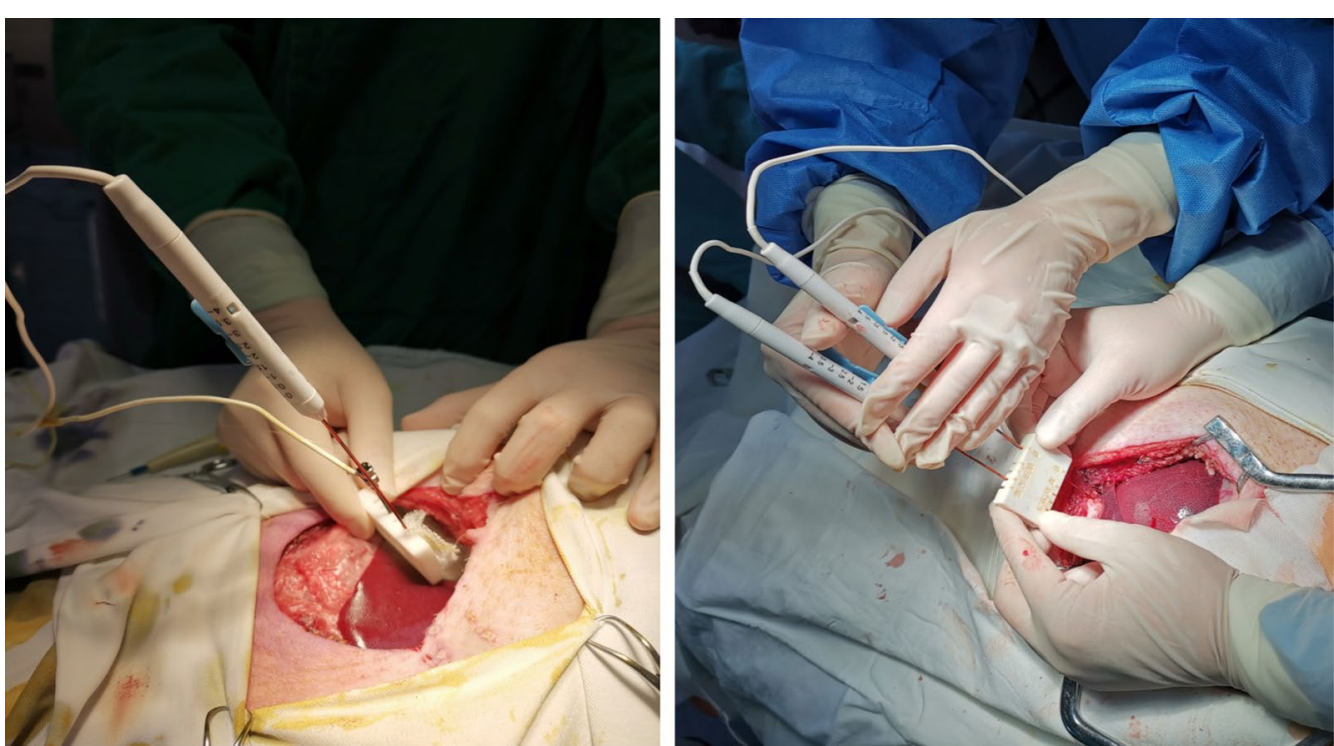

Fig. 1 Intra-operative image demonstrating liver placement of electrodes. Placement of IRE delivery device (left). Placement of H-FIRE device (right)
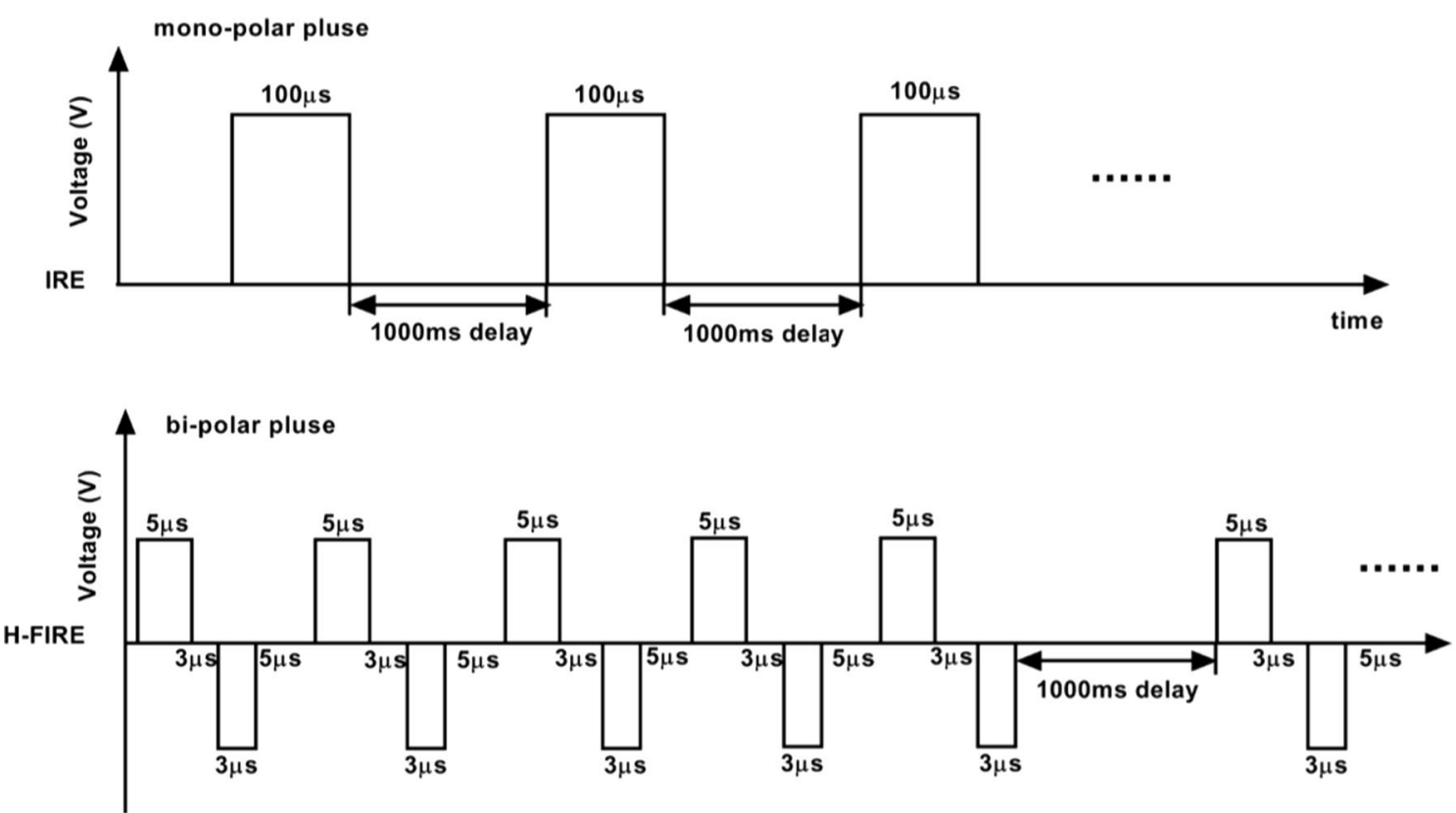

Fig. 2 Schematic representation of electrical pulse delivery using IRE (upper) and H-FIRE (lower)

IRE-treated, H-FIRE treated heart were fixed in formalin, and sliced for haematoxylin and eosin (H\&E) staining. The diagnoses of hearts were made by two senior attending doctors blinded in the pathology department.
Magnetic resonance imaging (MRI) imaging and analysis

All images were acquired using an abdominal coil (32-Channel abdominal Coil; GE Medical, Fairfield, USA) in a 3.0-T clinical MRI unit (GE, MAGNETOM $750 \mathrm{~W}$ $3.0 \mathrm{~T}$ ), with the animal in a prone or lateral position. 
Table 1 The parameters of IRE and H-FIRE ablation

\begin{tabular}{lll}
\hline & IRE & H-FIRE \\
\hline Number of pulses & 100 & 2400 \\
Voltage & $2200 \mathrm{~V}$ & $3000 \mathrm{~V}$ \\
Pulse width & $100 \mu \mathrm{s}$ & $5 \mu \mathrm{s}$ (postive) \\
& & and 3 $\mu \mathrm{s}$ (nega- \\
& tive) \\
Pulse direction & Unidirectional & Bidirectional \\
Pulse interval & $1000 \mathrm{~ms}$ & $1000 \mathrm{~ms}$ \\
Number of pulses in one group & 10 & 10 \\
Number of group & 10 & 240 \\
Positive and negative pulse interval & None & $3 \mu \mathrm{s}$ \\
\hline
\end{tabular}

Similar to prior studies [10], the ablation zone size was defined as the unenhanced region on contrast-enhanced MRI with reduced signal intensity as compared with normal liver tissue. Determine whether an effective ablation was performed.

\section{Histology}

Sections were cut at a thickness of $5 \mu \mathrm{m}$, and representative sections were stained with hematoxylin \& eosin $(H \& E)$. The diagnoses were made by a senior attending doctor in the Pathology Department.

\section{Statistical analysis}

Statistical analysis was performed using GraphPad Prism version 8 software (GraphPad Prism Software Inc., San Diego, CA, USA). Continuous variables are expressed as mean \pm standard deviation (SD). Categorical variables were summarized as numbers (\%). The abnormal changes of ECG between IRE group and H-FIRE group was compared using Fisher's exact tests. The change of cardiac biomarkers between IRE group and H-FIRE group was compared using t-tests. A $P$ value $<0.05$ was considered significant.

\section{Results}

IRE and asymmetrical waveform of H-FIRE performed effective ablation in this study

Gross tissue revealed an oval shaped ablation zone. Long axis of ablation zone was parallel to the emission electrodes (Fig. 3a, d). Following HE staining, the ablation area showed the normal liver structure was damaged, and instead of large areas of haemorrhage as well as inflammatory cell infiltration (Fig. 3b, e). Ablative lesions in liver tissue was evaluated with contrast-enhanced MRI. All lesions appeared as a focal or ovoid area. Ablation zone on contrast-enhanced MRI was with reduced signal intensity as compared with normal liver tissue (Fig. 3c, f).

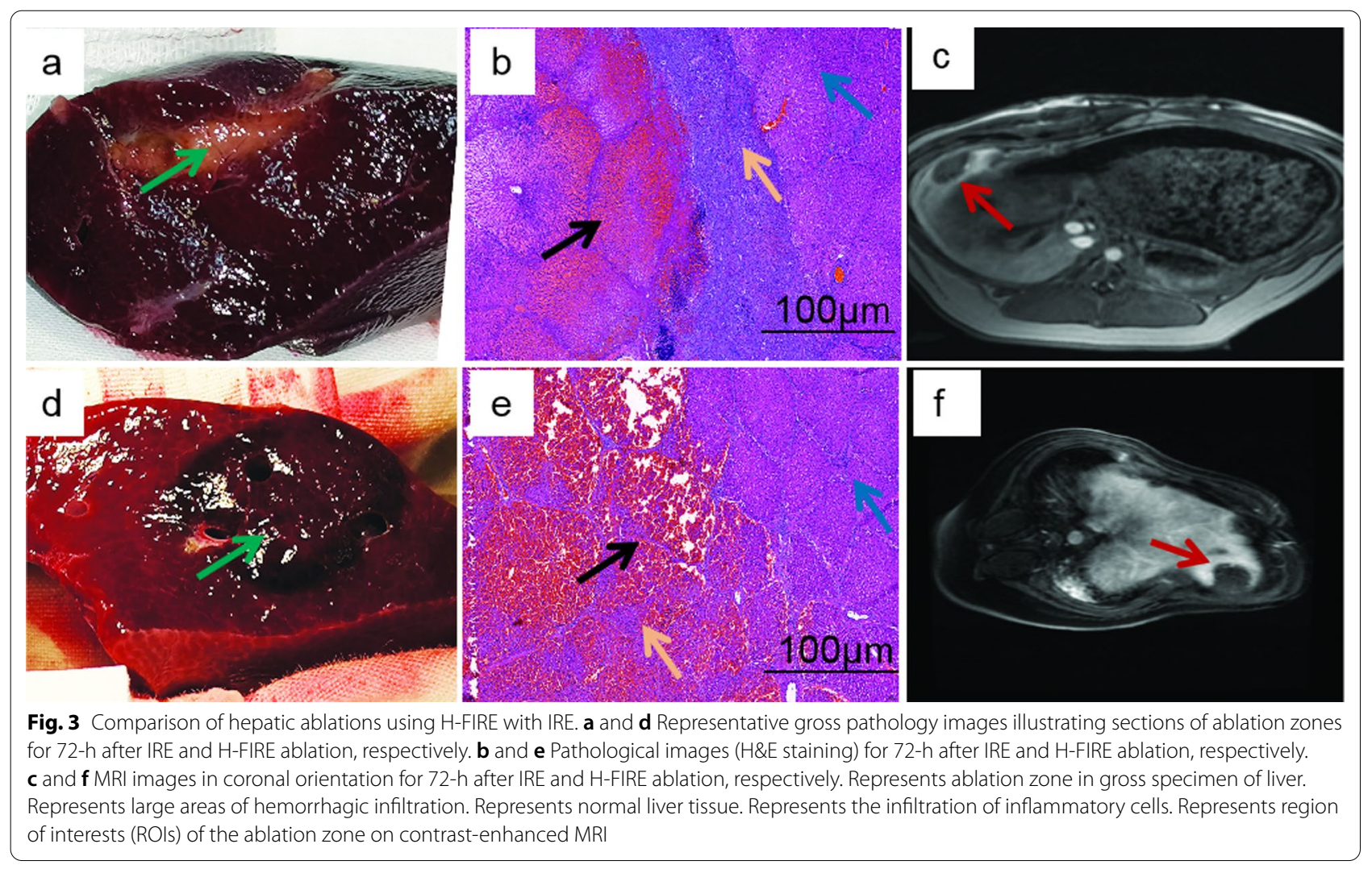




\section{The effect of the depolarization and repolarization} of ventricle was similar

Of the fourteen piglets, seven received IRE and the remaining seven received asymmetric waveform of H-FIRE. No matter where the electronic signal occurred, there was no significant changes in QRS waveform and QRS duration of the original sinus excitation. In this animal study, we did not notice a significant drop or elevation in myocardial voltage after IRE or H-FIRE. None of the piglets developed ST-segment deviations or T-wave changes related to the ablation of IRE or H-FIRE (Fig. 4). The current of each ablation are summarized in Table 2.

\section{The histology of heart accepted IRE and H-FIRE treatment were normal}

Both hearts tissue with IRE (7/7) and H-FIRE (7/7) treated were normal. There was no injure of heart can be seen (Fig. 5).

\section{Cardiac biomarkers demostrated the safety of H-FIRE}

Regarding cardiac biomarkers, IRE group showed an increase in cTnI after four hours of the ablation. However, the concentration of cTnI after $72 \mathrm{~h}$ of the ablation was lower to baseline. Whereas, the level of cTnI showed no significant change in H-FIRE group during the ablation (Fig. 6).

\section{None minor or major events of IRE and H-FIRE occurred} All animals survived and tolerated IRE or H-FIRE delivery until sacrifice. None of the 14 animals experienced a minor or major arrhythmic complication.
Table 2 The current in IRE and H-FIRE ablation

\begin{tabular}{lc}
\hline IRE (A) & H-FIRE (A) \\
\hline $14-16$ & $14-16$ \\
$15-18$ & $10-12$ \\
$10-12$ & $10-12$ \\
$11-15$ & $10-14$ \\
$12-13$ & $8-12$ \\
$12-14$ & $11-13$ \\
$13-15$ & $12-14$ \\
\hline
\end{tabular}

In IRE nad H-FIRE, the current fluctuates in a range. this table is reflect the range of current

\section{Discussion}

In the present study, we evaluated the cardiac safety of a novel asymmetrical waveform for H-FIRE, and compared it to IRE of the equivalent energy. In this study, the ablation efficacy of H-FIRE was similar to IRE of liver. IRE group showed an increase in cTnI after four hours of the ablation. However, the concentration of cTnI after $72 \mathrm{~h}$ of the ablation was lower to baseline. Whereas, the level of cTnI showed no significant change in H-FIRE group during the ablation. In consistent with previous study, these results were due to the maximum current and the intensity of energy release applied of IRE was higher than H-FIRE [11].

Cardiac depolarization and repolarization are depended on ion flux alteration inside and outside of the cardiac cells. External electrical stimuli can alter cell membrane potential, leading to a localized depolarization [12]. The fundamental of electroporation is permeabilization of the cell, which can induce cardiac arrhythmia [3]. Previous studies suggested that the current electricity

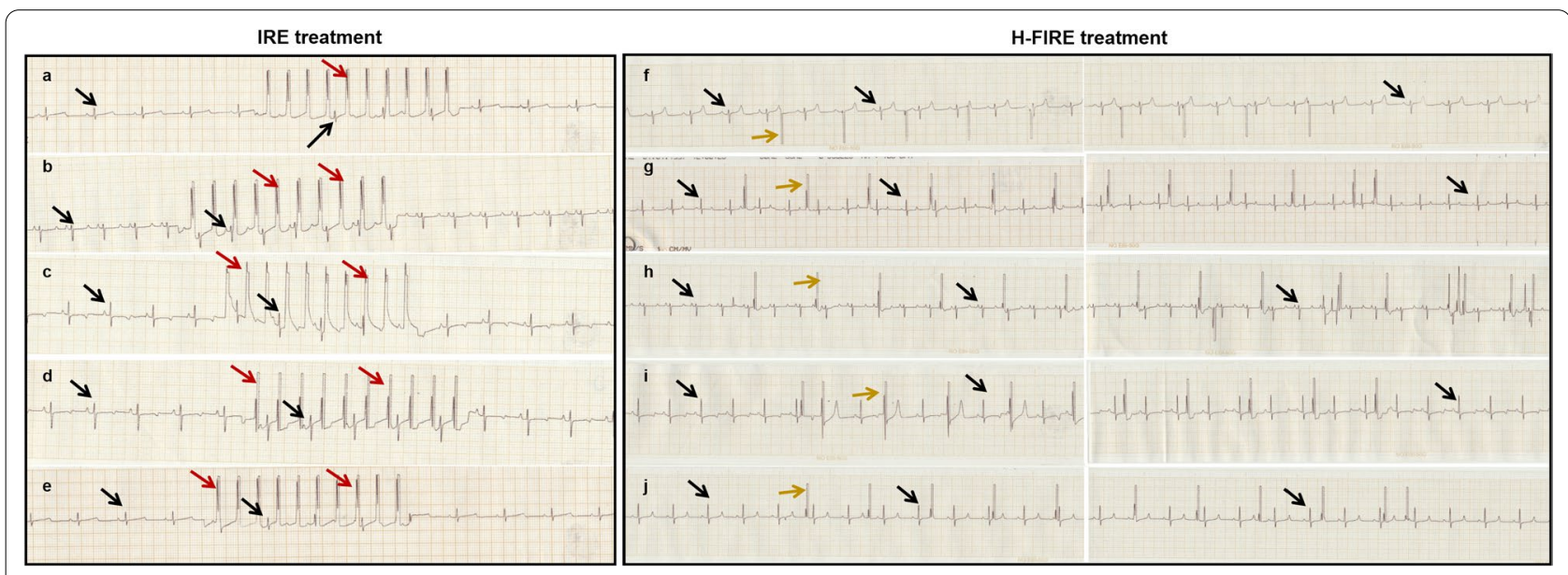

Fig. 4 ECG of five piglets in each group with the change of QRS waveform in the ablation procedure. $\mathbf{a}-\mathbf{e}$ ECG of five piglets during IRE ablation. $\mathbf{f}-\mathbf{j}$ ECG of five piglets during H-FIRE ablation. Represents autonomous cardiac rhythm. Represents electrical signal of IRE. Represents electrical signal of H-FIRE 

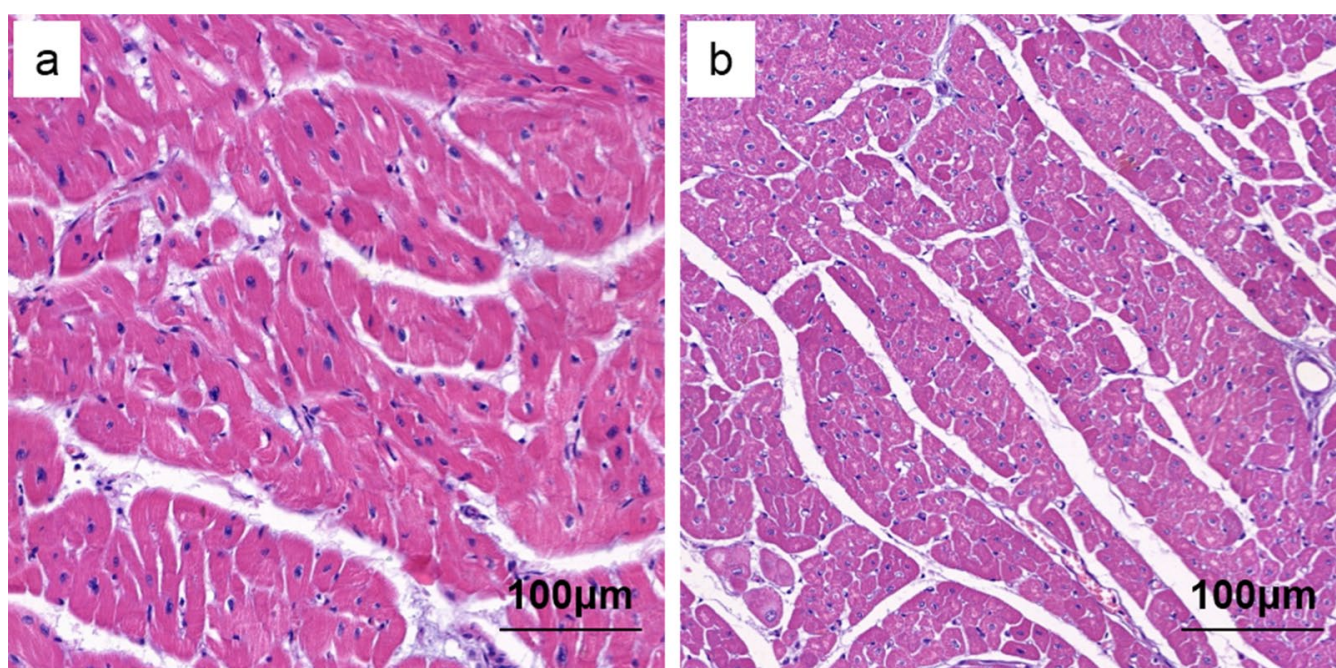

Fig. 5 Comparison the histology of heart with IRE and H-FIRE treated. a The histology of heart with IRE. b The histology of heart with H-FIRE. Both a and b was normal without myocardial injury

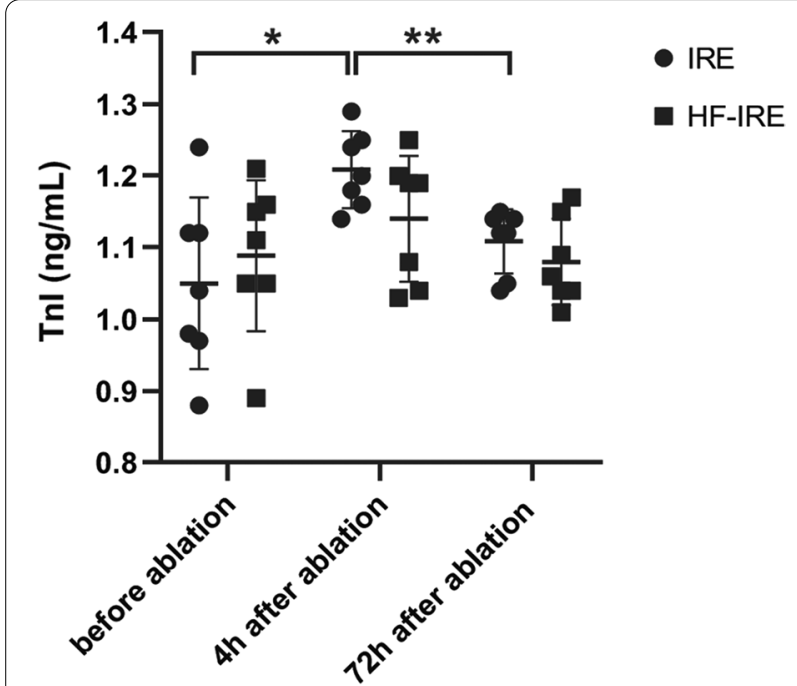

Fig. 6 Comparison of Tnl level using H-FIRE with IRE. The concentration of cTnl after $4 \mathrm{~h}$ of IRE ablation increased, then cTnl level decreased to baseline after $72 \mathrm{~h}$ of IRE ablation. cTnl level before and after H-FIRE ablation did not alter. ${ }^{*} P<0.05 ;{ }^{*} P<0.01 . n=7$

may trigger cardiac arrhythmia during and after IRE ablation near to the heart. Therefore, IRE was modified delivering electric impulses with ECG synchronization $[3,4]$. Different from IRE, H-FIRE delivers bipolar pulses of $1-5 \mu \mathrm{s}$ in a rapid burst with the benefits in reducing nerve and muscle stimulation [7]. These properties allow the administration of H-FIRE without the requirement of paralytics. Theoretically, it is speculated that H-FIRE obviates the requirement of cardiac synchronization.
There are two waveform for H-FIRE. Moreover, asymmetric H-FIRE is more effective than symmetrical H-FIRE in vivo [10]. Previous report showed that no EKG abnormalities during symmetrical H-FIRE ablating the hepatic tissue in swines [13]. Thus, specific safety issues on the heart with asymmetric H-FIRE should be discussed. It is well known that distance is key to avoid cardiac arrhythmia. Previous study of IRE suggested that synchronization may be necessary at distances greater than $1.7 \mathrm{~cm}$ from the myocardium (as measured on an axial CT scan) [5]. We speculated that the results in our study might due to the electric pulses on liver was a stimulus of remote field.

It is worthy that the effective refractory period was a fundamental prerequisite for the maintenance of fibrillation conduction. If electrical stimuli fall on the vulnerable period, it can induce cardiac malignant arrhythmia. In contrast, if electrical stimuli fall during the absolute refractory period, arrhythmogenic potential is minimized. Refractory period of the ventricular myocardium refers to the starting of QRS complex to the peak of $\mathrm{T}$ wave. Vulnerable period of the ventricular myocardium refers to the peak of $\mathrm{T}$ wave to the ending of $\mathrm{T}$ wave (Tpeak-Tend). In our study, most of the electrical stimuli detected in ECG arises on QRS complex of the swine's autonomous cardiac rhythm. Although H-FIRE pulse was arised on the vulnerable period, no existing dysrhythmia was seen of H-FIRE. ECG is the tracing of cardiac electrical activity. Electrical pulses of H-FIRE detected on ECG did not mean the stimuli conducted to the cardiac cell. It is the limitation of our study that the intra-cardiac electrical activity did not examine. We did not know 
whether the electrical signal of H-FIRE delivered on vulnerable period could cause cardiac arrhythmia. QT interval, QTc interval, and Tpeak-Tend interval are markers of cardiac arrhythmia. Both prolonged QT and QTc [14] and shortened QT interval and QTc [15] are associated with malignant ventricular arrhythmia (VAs). Prolonged Tpeak-Tend is associated with an elevated arrhythmic risk [16]. Compared with the ECG before H-FIRE ablation, QT interval, QTc and Tpeak-Tend did not change in the present study. These results suggested that no electrocardiogram abnormalities were observed during H-FIRE for asymmetrical waveform. Whereas, our results were contrast to a meta-analysis, which showed that the incidence of cardiac arrhythmias related to IRE on liver was 16\% (21 of 129 patients) [17]. Due to the patient with cardiac comorbidity was prone to cardiac arrhythmia. In addition, we found the piglets had an increase in cTnI after $4 \mathrm{~h}$ of IRE ablation. However, the concentration of cTnI decreased to baseline after $72 \mathrm{~h}$ of IRE ablation, indicating cardiac injury is temporary. Moreover, level of cTnI had no significant change during the administration of H-FIRE. That suggested H-FIRE maybe safe than conventional IRE. Symmetric H-FIRE is safer than IRE and asymmetric H-FIRE for the pluses cancelled each other out. But the ablation efficiency also could be impacted. Asymmetric H-FIRE is the balance between safe and efficiency.

Our study has many limitations. First, the number of animals used for this study was small. Second, whether stimulation on liver with asymmetrical H-FIRE has an effect on the heart, future studies on H-FIRE should incorporate measurements of intra-cardiac potential. Third, this study was based on normal porcine model, future studies about cardiac safety of H-FIRE needs to evaluate in patients with liver tumor.

\section{Conclusions}

In conclusion, these results showed H-FIRE with asymmetrical waveform seems relatively safe without major cardiac complications on the normal liver without tumors and the piglets without any potential cardiac diseases.

\section{Abbreviations \\ H-FIRE: High-Frequency Irreversible Electroporation; IRE: Irreversible elec- troporation; ECG: Electrocardiogram; cTnl: Cardiac Troponin I; ROI: Region of interests.}

\section{Acknowledgements}

Not applicable.

\section{Authors' contributions}

YYX conceived of the project and designed the study. JL modified the article according to the suggestions of reviewers. JL, XBZ, and XZ performed the trial. JJW analysed the data. JJW and JL wrote the manuscript. HMG and YYX edit the manuscript. All authors read and approved the final manuscript. $J L$ and YYX are the guarantors of this work and take responsibility for the contents of the article. All authors have contributed significantly and are in agreement with the content of the manuscript. All authors read and approved the final manuscript

\section{Funding}

This work was supported by the National Natural Science Foundation of China (Grant number: 81771944 to Yue yong Xiao).

\section{Availability of supporting data}

The datasets used and/or analyzed during the current study available from the corresponding author on reasonable request.

\section{Declarations}

\section{Ethical approval and consent to participate}

The study was approved by the Institutional Animal Care and Use Committee of The General Hospital of People's Liberation Army (License Number: 2019D15-07). The study was carried out in compliance with the ARRIVE guidelines. The study was conformed to the National Institutes of Health Guide for Animal Care and Use of Laboratory Animals (NIH Publication No. 85-23, revised 2011).

\section{Consent for publication}

Not applicable.

\section{Competing interests}

All the authors have no competing interests to declare.

\section{Author details}

${ }^{1}$ Department of Radiology, The First Medical Center of Chinese, PLA General Hospital, Beijing, China. ${ }^{2}$ Department of MRI, Affiliated Hospital, Logistics University of Chinese Peoples Armed Police Forces, Tianjin 300162, China. ${ }^{3}$ Department of Critical Care Medicine, Tianjin First Center Hospital, Tianjin 300192, China.

Received: 28 May 2021 Accepted: 29 November 2021

Published online: 07 December 2021

\section{References}

1. Neumann E, Schaefer-Ridder M, Wang Y, Hofschneider PH. Gene transfer into mouse lyoma cells by electroporation in high electric fields. EMBO J. 1982;1:841-5.

2. Garcia PA, Rossmeisl JH, Neal RE 2nd, et al. Intracranial nonthermal irreversible electroporation: in vivo analysis. J Membr Biol. 2010;236:127-36. https://doi.org/10.1007/s00232-010-9284-z.

3. Al-Khadra A, Nikolski V, Efimov IR. The role of electroporation in defibrillation. Circ Res. 2000;87:797-804. https://doi.org/10.1161/01.res.87.9.797.

4. Tovar O, Tung L. Electroporation of cardiac cell membranes with monophasic or biphasic rectangular pulses. Pacing Clin Electrophysiol. 1991;14(11 Pt 2):1887-92. https://doi.org/10.1111/j.1540-8159.1991.tb027 85.x.

5. Deodhar A, Dickfeld T, Single GW, et al. Irreversible electroporation near the heart: ventricular arrhythmias can be prevented with ECG synchronization. Am J Roentgenol. 2011;196:W330-5. https://doi.org/10.2214/AJR. 10.4490 .

6. O'Neill CH, Martin RCG, 2nd. Cardiac synchronization and arrhythmia during irreversible electroporation. J Surg Oncol. 2020;122:407-11. https:// doi.org/10.1002/jso.26041.

7. Arena CB, Sano MB, Rossmeisl JH Jr, et al. High-frequency irreversible electroporation (H-FIRE) for non-thermal ablation without muscle contraction. Biomed Eng Online. 2011;10:102. https://doi.org/10.1186/ 1475-925X-10-102.

8. O'Brien TJ, Passeri M, Lorenzo MF, et al. Experimental high-frequency irreversible electroporation using a single-needle delivery approach for nonthermal pancreatic ablation in vivo. J Vasc Interv Radiol. 2019;30(6):854862.e7. https://doi.org/10.1016/j.jvir.2019.01.032. 
9. Sano MB, Fan RE, Xing L. Asymmetric waveforms decrease lethal thresholds in high frequency irreversible electroporation therapies. Sci Rep. 2017;7:40747. https://doi.org/10.1038/srep40747.

10. van Es R, Konings MK, Du Pré BC, et al. High-frequency irreversible electroporation for cardiac ablation using an asymmetrical waveform. Biomed Eng Online. 2019;18:75. https://doi.org/10.1186/s12938-019-0693-7.

11. Kostrzewa M, Tueluemen E, Rudic B, et al. Cardiac impact of R-wave triggered irreversible electroporation therapy. Heart Rhythm. 2018;15(12):1872-9. https://doi.org/10.1016/j.hrthm.2018.07.013.

12. DeBruin KA, Krassowska W. Modeling electroporation in a single cell. I. Effects Of field strength and rest potential. Biophys J. 1999;77:1213-24. https://doi.org/10.1016/S000.6-3495(99)76973-0.

13. Siddiqui IA, Latouche EL, DeWitt MR, et al. Induction of rapid, reproducible hepatic ablations using next-generation, high frequency irreversible electroporation (H-FIRE) in vivo. HPB (Oxford). 2016;18:726-34. https://doi. org/10.1016/j.hpb.2016.06.015.

14. Dhutia H, Malhotra A, Parpia S, et al. The prevalence and significance of a short QT interval in 18,825 low-risk individuals including athletes. Br J Sports Med. 2016;50:124-9. https://doi.org/10.1136/bjspo rts-2015-094827.

15. Ye T, Zhang C, Wu G, et al. Pinocembrin decreases ventricular fibrillation susceptibility in a rat model of depression. Front Pharmacol. 2020;11:547966. https://doi.org/10.3389/fphar.2020.547966.

16. Opthof T, Coronel R, Wilms-Schopman FJG, et al. Dispersion of repolarization in canine ventricle and the electrocardiographic T wave: Tp-e interval does not reflect transmural dispersion. Heart Rhythm. 2007;4:341-8. https://doi.org/10.1016/j.hrthm.2006.11.022.

17. Scheffer HJ, Nielsen $\mathrm{K}$, de Jong MC, et al. Irreversible electroporation for nonthermal tumor ablation in the clinical setting: a systematic review of safety and efficacy. J Vasc Interv Radiol. 2014;25:997-1011. https://doi. org/10.1016/j.jvir.2014.01.028

\section{Publisher's Note}

Springer Nature remains neutral with regard to jurisdictional claims in published maps and institutional affiliations.

- fast, convenient online submission

- thorough peer review by experienced researchers in your field

- rapid publication on acceptance

- support for research data, including large and complex data types

- gold Open Access which fosters wider collaboration and increased citations

- maximum visibility for your research: over 100M website views per year

At BMC, research is always in progress.

Learn more biomedcentral.com/submissions 